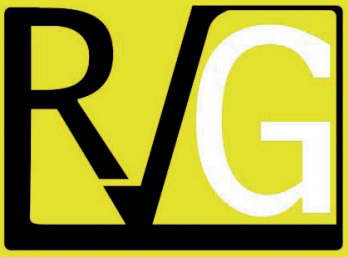

Julio - Septiembre, 2021

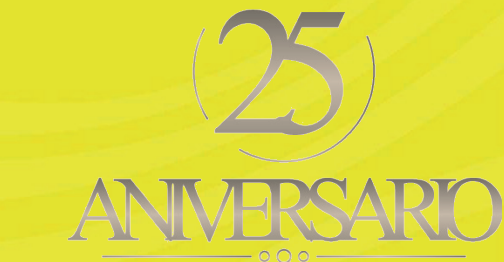

ANMERSARO
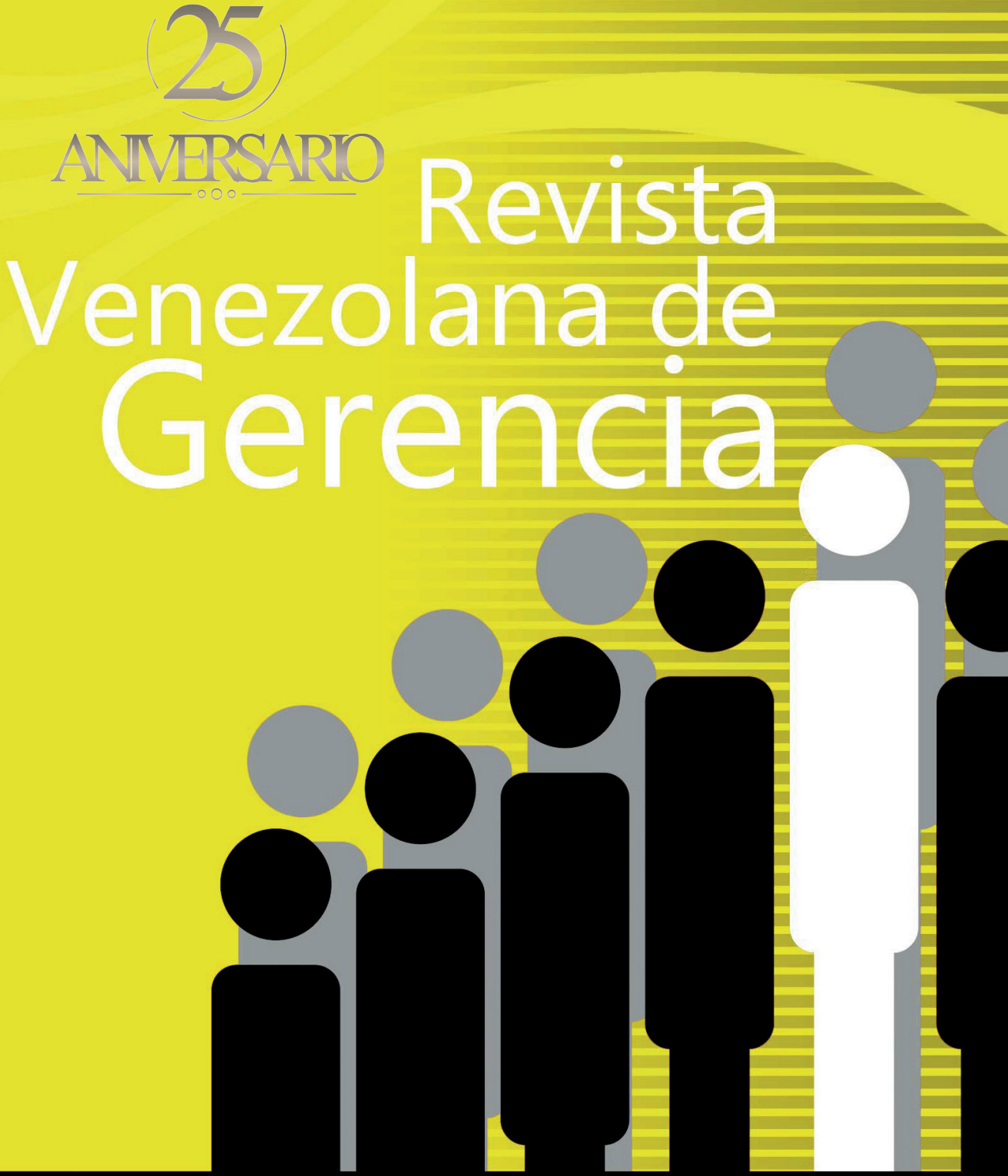

UNIVERSIDAD DEL ZULIA (LUZ)

Facultad de Ciencias Económicas y Sociales Centro de Estudios de la Empresa

ISSN 1315-99

Esta obra está bajo una licencia de Creative Comm Reconocimiento-NoComercial-Compartirlgual 3.0 Unpo http://creativecommons.org/licenses/by-nc-sa/3.0/deed.es 
COMO CITAR: Chuquihuanca Yacsahuanca, N., Pesantes Shimajuko, S. M., Vásquez Rodríguez, L., y Vargas De Olgado, E. C. (2021). Cultura digital desde el contexto universitario en tiempos de pandemia Covid-19. Revista Venezolana de Gerencia, 26(95), 802-817. https://doi.org/10.52080/
Universidad del Zulia (LUZ)

Revista Venezolana de Gerencia (RVG)

Año 26 No. 95 Julio-Septiembre 2021, 802-817

ISSN 1315-9984 / e-ISSN 2477-9423

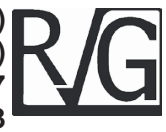

\title{
Cultura digital desde el contexto universitario en tiempos de pandemia Covid-19
}

\author{
Chuquihuanca Yacsahuanca, Nelson* \\ Pesantes Shimajuko, Soledad Marlene** \\ Vásquez Rodríguez, Luis*** \\ Vargas De Olgado, Elena Cachicatari ${ }^{* * * *}$
}

\section{Resumen}

El objetivo de la presente investigación consistió en reflexionar sobre la cultura digital desde el contexto universitario en tiempos de Pandemia Covid-19, que se ha manifestado en la era de la información producto de esta nueva realidad social. La investigación corresponde a una revisión teórica documental y/o bibliográfica. Los resultados expresan que a partir de las consideraciones actuales y el orden social -posmodernidad-, exigen que se revisen conceptos y prácticas que aún siguen imperando en la educación universitaria, inclusive desde sus cimientos. Existen reflexiones valorativas acerca de la incorporación de una andragogía socio constructivista como práctica educativa bajo el contexto de educación multimodal, donde se debe fomentar el diálogo, comunicación efectiva y las relaciones afectivas para el desarrollo de la cultura digital del docente universitario-investigador y de esta manera facilitar y contribuir en una transformación que fomente el desarrollo educativo integral del estudiante universitario. Se pretende establecerla postura del docente universitario bajo este nuevo contexto, donde se presenta una nueva realidad en la cual se aceleró con la aparición de la COVID-19.

Palabras clave: cultura digital; posmodernidad; educación multimodal; andragogía; educación universitaria.

* Licenciado en Educación, con maestría en docencia y gestión educativa de la universidad cesar vallejo, estudios de doctorado en Educación, ORCID: http://orcid.org/0000-0002-7354-2965

** Licenciada en enfermería, Doctora en Desarrollo de las ciencias sociales, Docente principal Universidad Nacional de Trujillo, Perú spesantes@unitru.edu.pe, ORCID: https://orcid.org/0000-0002-3015-9210

*** Docente Doctorado en Educación, Universidad Antonio Guillermo Urrelo, ORCID: https://orcid.org/00000002-2331-6337, Correo: luvaro78@hotmail.com

*** Universidad Jorge Basadre Gronhmann, Perú, Decana de la Enfermería de la Universidad Jorge Basadre Groghman. cachicatariv@unjbg.edu.pe ORCID: https://orcid.org/000-0000-9843-432X 


\title{
Digital Culture in the University Context: Value Reflections in Times of the Pandemic
}

\begin{abstract}
The objective of this research was to reflect on digital culture from the university context in times of the Covid-19 Pandemic, which has manifested itself in the information age as a result of this new social reality. The research corresponds to a theoretical documentary and / or bibliographic review. The results express that, based on current considerations and the social order -posmodernity-, they demand that concepts and practices that still prevail in university education be reviewed, even from its foundations. There are evaluative reflections about the incorporation of a socio-constructivist andragogy as an educational practice under the context of multimodal education, where dialogue, effective communication and affective relationships should be promoted for the development of the digital culture of the university teacher-researcher and of this $A$ way to facilitate and contribute to a transformation that fosters the integral educational development of the university student. It is intended to establish the position of the university teacher under this new context, where a new reality is presented in which it accelerated with the appearance of COVID-19.
\end{abstract}

Keywords: Digital culture; Postmodernity; Multimodal Education; Andragogy, University education.

\section{Introducción}

Uno de los factores que afecta directamente el crecimiento y desarrollo de los individuos y sociedades es la educación. La educación enriquece la cultura y creencias, y todo lo que caracteriza al ser humano; por tal razón es importante en todos sus sentidos, en virtud que transforma vida y genera cambios.

En el siglo XXI y en medio de una pandemia, el director general de la Organización Mundial de la Salud (OMS), Tedros Adhanom Ghebreyesus, declaró que el coronavirus COVID-19 pasa de ser una epidemia a una pandemia, afectando la posibilidad de continuar con una educación presencial a nivel mundial.

Es relevante enfatizar que la Organización de las Naciones Unidas (ONU), por medio de su organismo para la Educación, la Ciencia y la Cultura (UNESCO), ha generado la Agenda de Educación Mundial, que busca implementar hasta el año 2030 y en la cual se abordan temas como el acceso a la educación, la cobertura, las herramientas y su calidad en el mundo, 

pandemia

(Bagozzi,2020).

En consecuencia, a raíz de la situación pandemia de la COVID-19 ha obligado a cerrar instituciones educativas en todos sus niveles, y de esta manera, suspender las clases presenciales. Esta situación coyuntural trae consigo un cambio de esquemas o paradigmas educativos, en donde ciertamente algunas instituciones manejaban como cultura educativa la modalidad presencial de estudios, sin embargo, esta situación mundial de salud, estableció un nuevo esquema, en que quizás muchas instituciones, incluyendo docentes, estudiantes e incluso padres y representantes no estaban preparados por no tener arraigada una cultura digital, lo que se evidencia en deserciones tanto en docentes como en estudiantes.

En este contexto, todo parece indicar que se pudiera establecer un modelo de enseñanza híbrida que combina lo presencial con las alternativas digitales, tomando lo mejor de ambas modalidades de estudio. El contexto actual, obliga a superar el miedo a la "deshumanización" debido a que sería un riesgo si se pensara en reemplazar completamente las aulas con inteligencias artificiales o dispositivos tecnológicos. Las capacidades humanas son y seguirán siendo irremplazables, pero las tecnologías representan herramientas que permiten apoyar los procesos y desde luego conllevaría abrir un abanico de oportunidades para aprender y compartir nuestro aprendizaje.

Desde hace tiempo se ha venido hablando acerca de cómo se transita una nueva revolución industrial, aún más arrolladora que las anteriores en virtud que no solo trasformará lo que se hace sino lo que existe. Se trata de la fusión de esferas entre lo tecnológico y andragógico a través de una mediación socio constructivista de los docentes. En este sentido, se sitúa al sujeto en el centro de la cosmovisión, porque reconoce que los conocimientos están hechos por y para los humanos.

De este modo, cuando los docentes diseñan espacios de aprendizaje, primero se reflexiona acerca del lugar que ocupa la persona que aprende en la experiencia de aprendizaje, su papel y motivación, la manera cómo se acercará a los objetos de aprendizaje, qué debe darse para promover una participación activa y otros principios fundamentales que requiere esta modalidad.

Esta visión andragógica concede importancia a las interacciones sociales, que condicionan cómo se construyen los conocimientos individuales sobre el mundo. De este modo, se reconoce que se aprende en grupo y sociedad. Es decir, resulta una visión histórico-social que considera cómo, bajo la presión de ciertos factores (económicos, sociales, políticos y culturales), las sociedades se desarrollan y evolucionan (Fourez, 2008:17).

En virtud de ello, para hacer frente a esta incertidumbre no prevista, los gobiernos y estados, conscientes de esta situación, buscan mecanismos para que la educación en todos los niveles siga estando activa, dando respuesta a miles de estudiantes y esta posible solución incorpora la modalidad educativa de educación a distancia o asistida por las tecnologías de información y comunicación (TICS), de acuerdo a lo expresado por Bartolomé (2008), quien expresa que una educación semi presencial hace referencia a una estrategia educativa basada en el uso intensivo de las nuevas tecnologías, estructuras operativas flexibles y métodos pedagógicos altamente 
eficientes en el proceso enseñanza y aprendizaje; son las que permiten que las condiciones de tiempo, espacio, ocupación o edad de los estudiantes no sean factores limitantes o condicionantes para el aprendizaje.

La combinación de esta nueva revolución industrial y el contexto de pandemia COVID-19, acelerarán los cambios en las habilidades que son consideradas fundamentales para adaptarse a los cambios. Esto hace que se planteen ciertas reflexiones valorativas que deben tomar en consideración los docentes para hacerle frente a los desafíos a nivel educativo y laboral en la sociedad actual, especialmente para los jóvenes. Se necesita acompañarlos adquirir habilidades para conseguir $y$ desarrollarse en empleos que todavía no se han creado, en virtud que las nuevas tecnologías también generarán diferentes formas de trabajo. Muchas de estas habilidades son ya imprescindibles en la actualidad mientras que otras van ganando terreno.

La investigación corresponde a una revisión teórica documental y/o bibliográfica de carácter deductivo, en virtud que consiste en un análisis de la información sobre un determinado tema, con el propósito de establecer relaciones, diferencias, etapas, posturas o estado actual del conocimiento, respecto a la temática objeto de estudio.

\section{Posmodernidad en la Educación: una corriente filosófica caracterizada por la modernidad y la ilustración.}

La filosofía posmoderna se puede expresar como una corriente filosófica que asume que se han sobrepasado las ideas que han caracterizado a la modernidad y a la llustración, en otras palabras, se mantienen en común una postura de crítica, desconfianza y libertad e incluso ruptura con las tradiciones ideológicas de la modernidad occidental. La posmodernidad descansa en los ideales de la razón y el progreso, un progreso cada vez más logrado por medio de la ciencia y la técnica.

Desde la perspectiva anterior, la posmodernidad tiene una vinculación con el posindustrialismo y con todo el compendio de ideas innovadoras y creativas. La cultura de la sociedad avanzada pasó por un profundo cambio en relación con la estructura y al pensamiento.

La riqueza de una potencia no descansa únicamente en la abundancia de materia prima, sino en la cantidad/ calidad de la información técnicocientífica que esta pueda generar. A la ciencia, en cuanto al modo de organizar, almacenar y distribuir información, le corresponde mostrar que sin el saber científico y técnico no se tiene riqueza, y la universidad, institución importante en el cálculo estratégico dogmático del Estado, asume la investigación tanto financiada como la de relevancia social para generar conocimiento a través de la enseñanza y la investigación, funciones históricas de la universidad, que antes buscaban formar hombres y mujeres para descubrir la verdad, hoy en día buscan en su mayoría, principalmente, formar individuos competentes para insertarlos en el mercado mundial.

El momento histórico por el cual atraviesa la humanidad es de gran significado para el hombre actual, la transición entre dos períodos, uno que está llegando a su fin (modernidad) y otro que se inicia (postmodernidad). Trae cambios asociados en el plano político, económico, social, cultural, 

pandemia

educacional, entre otros. Requiere un cambio paradigmático en la vida del hombre. Actualmente se vive en un mundo y una sociedad multicultural, donde los medios de comunicación social ofrecen una pluralidad de formas de vida, modos de pensamiento y toda clase de información en la que, todo o casi todo hoy vale con tal que alguien o algunos lo respalden, como ejemplo de ello son las informaciones generadas por las redes sociales.

Dentro de este aspecto, el crecimiento exponencial del conocimiento e información, el desarrollo tecnológico y científico, el vertiginoso avance de las telecomunicaciones que permite estar conectados casi instantáneamente con otro punto del ámbito global. A través de la red (Internet) es posible realizar transacciones económicas, adquirir información, realizar estudios a distancia, ofrecer productos de consumo masivo, entre otros.

La forma en la cual nos comunicamos ha cambiado en 180 grados comparado con otros períodos de la humanidad, en donde el sector educativo motivado a la pandemia en la que se encuentran el uso de estas herramientas tecnológicas han constituido una opción para afrontar y hacerle frente a este desafío, en la que <<obliga>> a los docentes y estudiantes a mediar con dichos recursos tecnológicos para lograr los objetivos académicos.

La postmodernidad educativa se inspira en la obra de Nietzsche, propugna desde el pensamiento, el impacto de la tecnología y la filosofía de sistemas, una nueva concepción del hombre. La postmodernidad es pues una filosofía antihumanista y en consecuencia, individualista que en el plano de la educación se dirime en la hegemonía de la tecnología, en la importancia del saber y del conocimiento en la sociedad del futuro y en la necesidad de la innovación permanente, lo cual se refleja en el uso de las herramientas tecnológicas, donde los valores humanos son puestos a prueba en este plano ontológico de acción, donde las emociones expresadas en el respeto, responsabilidad, empatía y solidaridad deben ser proyectadas y extendidas con el uso de dichas tecnologías, las relaciones sociales y afectivas deben prevalecer para la constitución de un nuevo paradigma educativo, donde la convivencia y colaboración entre los individuos deben estar en función de un aprendizaje compartido y social.

En la posmodernidad, no obstante, Lyotard (2004:25) establece que la hegemonía del poder de la ciencia, que se encuentra en estado de deslegitimidad y que tiene un saber parcial, se opone el saber narrativo. Por lo que, la ciencia está hecha de enunciados denotativos, el saber comprende el saber-hacer, el saber-vivir, el saber oír, entre otros.

Desde la perspectiva anterior, se puede decir que el saber ha sufrido trasformaciones muy evidentes, tanto es así que, en los actuales momentos tiene la forma de información, y esta a su vez tiene valor de cambiar. Con la introducción generalizada de los computadores en la sociedad y en la cultura todo se determina por su operatividad y su eficacia instrumental. También el saber que, en este sentido, tomará el cuerpo de una mercancía o, dicho con más precisión, se convertirá en mercancía, en objeto intercambiable regido por la lógica de la compra y la venta. De allí que (Lyotard, 1989:150) explica que no es posible interpretar al mundo sin alterarlo:

(...) se trata de transformarlo (...):

la humanidad solo se plantea los 
problemas que está en condiciones de resolver (...). Si hay que transformar el mundo es porque él mismo se está transformando. (...). Si es verdad que el mundo pide ser transformado es porque hay un sentido en la realidad que pide acontecer.

Desde esta postura, la postmodernidad en el ámbito educativo evidencia en los actuales momentos transformaciones propiciadas por varios factores externos (globalización, situación de pandemia, aspectos sociales, políticos y económicos, entre otros) e internos (cambios en el currículo educativo, planes de estudios, roles del docente y del estudiante, entre otros), donde se presenta desde los cimientos realidades que ameritan ser cambiadas o alteradas para lograr el encauzamiento educativo dentro del nuevo orden social, económico y político.

\section{Cultura Digital en el contexto educativo: fomento y manejo de herramientas tecnológicas.}

Desde el contexto de la pandemia actual, las instituciones educativas en todos los niveles han experimentado una alteración de las rutinas educativas, donde estaban arraigadas en su mayoría por clases presenciales, donde los docentes y estudiantes a pesar del manejo de herramientas tecnológicas de manera cotidiana, tuvieron que cambiar sus actitudes y maneras tradicionales de acceder a la educación, por ende Camacho, et al. (2020) señala que estos elementos fueron influyeron de forma significativa en el desenvolvimiento de empresas, organizaciones y sistemas, entre ellos el educativo, rompiendo paradigmas en el proceso de enseñanzaaprendizaje.

Lo antes expuesto, establecen nuevas formas de enseñar y formas de aprender a través de la tecnología educativa; cobra mucha fuerza la cultura digital que se debe establecer en este momento, y se debe mantener luego de la coyuntura actual del sistema de salud.

La cultura digital es un concepto general que describe la idea que la tecnología e Internet configuran significativamente la forma en que los individuos interactúan, se comportan, piensan y se comunican como seres humanos en un entorno social-digital (Cabero, 2014), en otras palabras, es el producto de la tecnología penetrante y el acceso ilimitado a la información desde cualquier sitio y en cualquier momento, resultado de la innovación tecnológica disruptiva en la sociedad.

En el ámbito educativo, la cultura digital cobra un gran auge en los actuales momentos, en virtud que representan el conjunto de prácticas, costumbres y formas de interacción social que se llevan a cabo a partir de los recursos de la tecnología digital como el Internet, y en las cuales los centros educativos (escuelas, liceos y universidades), desde hace años debieron implementar con mayor ímpetu, para que esta cultura digital en los actuales momentos estuviese fortalecida para afrontar la situación coyuntural de pandemia a través de la COVID-19, que se presenta en la actualidad.

Para nadie es un secreto que la cultura digital debe ser cultivada para que esta quede fortalecida y pueda brindar un mejor aprovechamiento de las clases a distancia, no solo en los estudiantes sino en sus docentes, que son los principales protagonistas de este proceso educativo, en virtud que son los garantes de la supervisión educativa del proceso de enseñanza y aprendizaje (Suárez et al. 2010). 

pandemia

La cultura digital incluye, a su vez, los nuevos medios que nacieron en el ámbito digital, como las reconstrucciones de la realidad virtual, el arte por Internet y las nuevas narraciones interactivas. También, las versiones digitales, los contenidos $y$ las funciones de las instituciones patrimoniales (bibliotecas, museos y archivos). Al igual que los museos y las instituciones patrimoniales, la cultura digital asume el reto de coleccionar, contextualizar, conservar y transmitir dicha información de una manera que permita recrear y compartir la información y de esta manera reproducir un nuevo conocimiento, a través de un pensamiento divergente, donde se propicien espacios de encuentros virtuales: comunidades de aprendizajes, en función de socializar la información y crear redes semánticas digitales (Salinas, Darder y De Benito, 2015).

Cabe señalar, que al hablar de "cultura digital", se destacan los "contenidos" al mismo nivel que los "medios", por muy novedosos que estos sean. Las "nuevas tecnologías", que ya no son "nuevas" a pesar del acelerado desarrollo de nuevos programas $y$ soportes tecnológicos, ocupan un lugar paralelo frente a la necesidad de contenidos de la industria hipermedia. De hecho, es el propio medio, con su lenguaje específico de integración y de interactividad, el que impulsa a crear nuevos contenidos acordes con dicho lenguaje.

Los docentes son los llamados a constituir, configurar, seleccionar e incluso crear y recrear dichos contenidos adaptado a las necesidades y exigencias de la demanda educativa actual. Muchas veces se encuentran con docentes, que mantienen las mismas metodologías tradicionales cuando usan las herramientas tecnológicas, y ocurre una desmedida incongruencia, es allí donde se requiere docentes preparados no solo técnicamente sino pedagógicamente, capaces de mediar didácticamente con las herramientas más apropiadas, proponiendo estrategias innovadoras que permitan formar a los estudiantes de manera efectiva, independiente y autónoma, colaborativamente y con sentido social (Freire,1997).

La cultura digital no sólo se debe considerar por el impacto de la introducción de las tecnologías en la enseñanza y en la transmisión de dicha cultura, que en muchos casos marcada por cierto determinismo tecnológico, sino por el estudio profundo del desarrollo de nuevos contenidos, esto no es más que la constitución, revisión, reestructuración y actualización del currículo educativo, en una adecuada calidad vinculada a los nuevos medios y a su diseño. Desde esta perspectiva, puede decirse que la brecha digital denunciada por la UNESCO no se solucionará simplemente con un despliegue tecnológico, pues prevalecerá la fractura cognitiva si no se desarrolla un esfuerzo paralelo en los contenidos adaptados al medio digital (Bindé, 2005:23).

Ahora bien, se puede decir que los seres humanos están utilizando tecnología moderna para mejorar o alterar la calidad de vida para adaptarse a nuestro entorno cambiante y las necesidades humanas. Por ejemplo, se puede observar, que en los actuales momentos se ha creado la economía digital, en la cual, se pueden adquirir los bienes y servicios que se necesitan de forma instantánea y la computación en la nube para trabajar sobre la marcha. $A$ medida que en nuestras vidas se vuelven cada vez más rápidas, se innova para adaptarse y debido a que la tecnología 
no se detiene, en palabras más simples: con el paso del tiempo, la tecnología tiende a multiplicar su rendimiento y a dividir su coste, en consecuencia, se tiene que compensar desarrollando la cultura digital según sea necesario.

La propia utilización de la convergencia digital de las Tecnologías de la Información y Comunicación (TICS), en el campo educativo y cultural, es un camino hacia la construcción de la educación y cultura digital en virtud que puede representarla según todos los valores tecnológicos que conlleva su resultado final. Es con las TICS y su convergencia digital que el ser humano tendrá o deberá aprender a organizarse de otra manera para incluir la educación y cultura digital como parte de sus vidas $y$, por otra parte, todas las instituciones sociales tendrán o deberán prepararse para incluir mejor y de modo más adecuado este proceso tecnológico. Desde este contexto, se resalta la intervención del estado y las entidades educativas gubernamentales, como entes garantes de establecer políticas públicas dentro del ámbito educativo que permita la concreción e instrumentación de las tecnologías dentro del sistema educativo con el compromiso $y$ pertinencia social-educativo-tecnológico.

Por ello, hoy día se entiende por educación y cultura digital aquel mundo de procesos, actividades, bienes 0 servicios que se generan en torno al uso de las TICS, y obviamente de su convergencia digital, la cual tiene como objeto apoyar el acceso a competencias cognitivas e innovadoras en procesos pedagógicos o culturales de aprender aprendiendo o hacer creando, y dentro de un círculo o proceso de formación y creación continua o permanente.

\section{Andragogía Socio constructivista: creación y construcción de conocimiento}

En general, un enfoque socio constructivista destaca los contextos sociales del aprendizaje, donde se resalta que, el conocimiento se crea y construye mutuamente, desde este contexto, se puede decir que la teoría socio constructivista de Vygotsky considera al individuo como ser social inmerso en un contexto sociohistórico.

Desde la perspectiva de Piaget, en contraposición a la de Vygotsky se observa un cambio conceptual desde el individualismo hasta la colaboración, interacción social y la actividad sociocultural. Según el enfoque cognitivo constructivista de Piaget, los estudiantes construyen el conocimiento al transformar, organizar y reorganizar los conocimientos previos y la información. (Wertsch,1988). El enfoque constructivista social de Vygotsky destaca que los estudiantes construyen el conocimiento a través de las interacciones sociales con los demás. El contexto de este conocimiento se ve influenciado por la cultura en la que vive el estudiante, que incluye la lengua, creencias y habilidades (Serrano y Pons, 2011).

Por otro lado, Piaget destaca que los facilitadores deberían ayudar a los discentes a explorar y desarrollar su comprensión, mientras que Vygotsky subraya que los facilitadores deberían ofrecer oportunidades a los discentes para que aprendan a construir el conocimiento, en colaboración con el facilitador y sus homólogos, (Wiske, 1999). En ambos modelos, los facilitadores son formadores y guías, más que directores y moldeadores 
del aprendizaje. Desde la perspectiva anterior, el socio constructivismo, entendido desde una perspectiva andragógica, puede definirse por varios rasgos característicos según Jonnaert (2002), entre los cuales se resalta los siguientes:

Uno de los rasgos que contempla es la construcción del conocimiento, es decir, el individuo es el protagonista en la construcción de su propio conocimiento. Estas ideas, imágenes, conceptos, teorías, abstracciones, entre otras, se recrean en la mente de cada persona, en otras palabras, se construye el conocimiento nuevo en la medida en que la persona interactúa con su entorno, pero el éxito y alcance de dicha construcción viene condicionado por las características neurológicas y biológicas de cada individuo, (Perkins, 2003).

En este sentido, los docentes se presentan como los principales protagonistas que deben garantizar a través de estrategias innovadoras en el contexto de la educación a distancia un aprendizaje autónomo, independiente y para toda la vida para sus estudiantes, donde el medio tecnológico constituye la principal herramienta para la mediación didáctica del proceso de enseñanza y aprendizaje. Es por ello, que el docente debe tener, principalmente una formación técnica que le permita hacer frente a estos desafíos y segundo una formación andragógica que permita establecer una mediación didáctica en este contexto de virtualidad.

En cuanto al rasgo que conforma la dimensión individual, la cual implica que los procesos cognitivos de rango superior como el pensamiento lógico o la memoria tienen lugar en el propio estudiante (Miller, 2011; Pritchard, 2013). La percepción que éste tiene de su entorno no sólo es individual sino también subjetiva. Es decir, la percepción que un grupo de individuos tiene de una misma realidad no siempre será la misma; incluso la reacción que una determinada situación o contexto puede provocar en un grupo de personas puede variar sustancialmente (Fourez, 2008).

Desde esta perspectiva, en el ámbito educativo, los docentes como protagonistas de este proceso de enseñanza y aprendizaje, deben adaptar los contenidos, estrategias e incluso los objetivos de aprendizaje a las necesidades y estilos de aprendizajes individuales de cada estudiante y de esta forma que los recursos tecnológicos $y$ estrategias instruccionales sean coherentes y pertinentes, sobre todo aprovechables por los estudiantes.

En cuanto, al rasgo perteneciente a la dimensión social, que entiende que si bien parte de la construcción del conocimiento se produce en el interior del individuo, no puede desligarse de su interacción social con su entorno. Esto significa que, se trata de un proceso de "creación y transferencia de lo externo a lo interno, de lo social hacia lo psicológico" (Medina, 2006: 215). Todos los procesos cognitivos de índole superior (atención, memoria, lenguaje, entre otros.) son inicialmente individuales; sin embargo, están socialmente conectados y no pueden desligarse. Es decir, que tanto esta actividad psíquica como la voluntad que ejerce el individuo sobre ella están "instrumentadas, estructuradas y transformadas en procedimientos (o herramientas) socialmente elaborados (como el lenguaje y todos los demás sistemas de signos que sirven para sentar y señalar relaciones entre objetos o propiedades de los objetos representados)" (Gaonac'h y Golder, 2005: 142). 
En relación a la concepción anterior, existe una sinergia entre el conocimiento adquirido y el aprendizaje para lograr ese conocimiento, donde las formas para llegar a este último, se transforman y evolucionan a través de la interacción con los individuos y su entorno social. Este proceso bidireccional es lo que permite al individuo construir conocimiento nuevo a partir del conocimiento previo. Esa percepción subjetiva de la realidad anteriormente mencionada tiene, sin embargo, un considerable componente social. Aunque el individuo trata la realidad de manera individual, sus propias interpretaciones están condicionadas por la interpretación socialmente dominante (Gaonac'h y Golder, 2005).

En cuanto al rasgo presente en la dimensión contextual, implica la interacción entre iguales, la cual contribuye a la construcción de conocimiento, resalta la importancia en el contexto en donde se desenvuelve el individuo, lo cual lo afecta de manera directa. De nuevo, se trata de un proceso bidireccional, dinámico: la persona aprende de y gracias a su entorno en el cual está inmerso, y es capaz de influir en él y modificarlo para producir un nuevo conocimiento. Cabe señalar que, en la medida en que el entorno cambia también modifica la influencia que ejerce sobre el individuo (Morales, 2010).

De modo que es capaz de crear conocimiento nuevo dependiendo de dicho entorno, en virtud que le permitirá de manera cognitiva internalizar dichos aprendizajes y establecer nuevos mecanismos para la activación de ese nuevo conocimiento que se recrearán en sus acciones y en actitudes, porque son capaces de transformar y profundizar sus pensamientos e ideas. En consecuencia, se puede decir que es un proceso que se da de forma permanente y no siempre conscientemente. La capacidad de representación que posee el individuo viene definida por su interacción con el entorno y la dimensión colectiva de su existencia (Medina, 2006).

Es así como, la evaluación es un proceso dinámico y social, es decir, la interacción entre docente y estudiante como parte indisoluble de la propia evaluación. No se trata de un proceso unidireccional, que tiene lugar al final de un período concreto y que termina en el momento en el que el docente asigna una nota o valoración a un determinado desempeño. Al contrario, se trata de una dinámica que se da a lo largo del proceso, que se modifica en función de la interacción entre docente y estudiante. Lo que el segundo aporta se modificará en función de las observaciones del primero; en correspondencia, las aportaciones del docente irán variando en función de la evolución del desempeño. Este proceso se produce desde el inicio mismo de la unidad didáctica, en virtud que se considera parte integrante de la misma y fuente irremplazable de aprendizaje (García-Valcárcel, 2013).

\section{Educación Multimodal y el Conectivismo Educativo: herramientas de enseñanza tradicional.}

En las últimas décadas ha existido un gran desarrollo en la tecnología digital y móvil. Los cambios en las nuevas tecnologías han llevado consigo cambios en los modos de comunicación y también en la definición de sus componentes y sus aplicaciones en el siglo $\mathrm{XXI}$ en los contextos educativos (Kress, 2010). En este nuevo contexto 
Chuquihuanca Yacsahuanca, Nelson; Pesantes Shimajuko, Soledad Marlene; Vásquez Rodríguez, Luis y Vargas De Olgado, Elena Cachicatari

Cultura digital en el contexto universitario: reflexiones valorativas en tiempos de pandemia

en que todo cambia rápidamente hay herramientas de la enseñanza tradicional que se mantienen, sin embargo, los nuevos tiempos demandan cambios en la enseñanza educativa en todos los ámbitos, de modo que las oportunidades de aprendizaje que ofrecen las nuevas tecnologías se incorporan en el proceso de enseñanza y aprendizaje.

La educación multimodal, se asume como un estilo de enseñanza y aprendizaje que se da cuando se utiliza el conjunto de los cuatro tipos de aprendizaje principales (visual, auditivo, kinestésico y de lectoescritura). Esto es debido a que no tiene definida ninguna preferencia a la hora de aprender, (Mayer, 2009). Por su parte, Covarrubias (2021) señala que "las instituciones educativas han buscado esa modernización de los contenidos con la inserción de las TIC's"

Muchos docentes ya han estado incorporando elementos multimodales a sus prácticas docentes de manera intuitiva, es decir, con más de un modo de comunicación, por ejemplo, textos en los que aparece imagen además de texto escrito, tal y como señala Knox (2008:140):

Perspectivas multimodales sobre el lenguaje y la educación han aparecido recientemente en la literatura sobre la enseñanza y aprendizaje. Una breve consideración de las prácticas en el aula de profesores y estudiantes. Sin embargo, muestra muy rápidamente que la multimodalidad es algo que los profesores han entendido y realizado intuitivamente durante mucho tiempo.

El aprendizaje enmarcado en esa ciencia clásica consideraba el "saber todo de todo" en un escenario particular y en base a las propias experiencias del individuo, sin considerar ningún tipo de relación socializante. Posteriormente, el constructivismo, como modelo emergente, tomó en consideración que el aprendizaje debe ser dinámico y motorizado por el propio aprendiz quien, en un ambiente de intercambio de experiencias, construye su propio conocimiento. Luengo, Luzón y Torres (2008; 28) así lo consideran cuando señalan que en la aprehensión de saberes deben estar presentes "habilidades cognitivas y prácticas, así como componentes sociales y de comportamiento no cognitivos, tales como actitudes, emociones, valores y motivación" y de esta manera se fomenta el desarrollo de las inteligencias múltiples de los individuos (Gardner, 2001).

Sin lugar a dudas, en el escenario mundial han surgido diversas teorías o modelos que, en cierta medida, tratan de explicar como ocurre el aprendizaje en esta nueva época conocida como era postindustrial o era del conocimiento utilizando como herramienta las nuevas tecnologías de información y computación. Una de las más resaltantes la constituye el conectivismo, la cual, según su autor Siemens (2006) "como teoría presenta un modelo de aprendizaje que refleja una sociedad en la que el aprendizaje ya no es una actividad individual".

Este tipo de aprendizaje, como señala Santamaría (2006:123), es el proceso de creación de redes. Los nodos son entidades externas que se pueden usar para crear una red. Los nodos pueden ser personas, organizaciones, libros $\mathrm{O}$ cualquier otra fuente de información. El acto de aprender consiste en la creación de una red externa de nodos, en la que se conecta y modela información y fuentes de conocimiento.

Desde este contexto, el conectivismo plantea diversos puntos de vista que convergen en diferentes 
aspectos o consideraciones de las teorías clásicas del aprendizaje, como la cognición y motivación; del pensamiento complejo, al considerar al constructivismo como modelo de intercambio social; de la teoría del caos, al señalar que la información no llega preformada sino en forma caótica o desordenada; de las nuevas tecnologías de información y computación, al considerar que la información se encuentra en las redes. De esta manera, esta última teoría del aprendizaje señala una apropiación del conocimiento por parte del individuo en un ambiente social donde el intercambio de saberes o experiencias es compartido por una diversidad de individuos, con la guía del instructor, a fin de crear y aprehender el conocimiento a través del uso de la tecnología.

\section{Reflexiones Finales}

Al comienzo del artículo se señaló la necesidad de un cambio paradigmático en el ámbito educativo destinado a construir un "individuo nuevo"; con ciertas competencias y manera de insertarse de la mejor manera posible en la sociedad de esta era tan compleja, en todos los sentidos, donde se cuestionan casi todos los conocimientos de los diferentes ámbitos del saber.

El discurso posmoderno, con el caos y la complejidad como referentes fundamentales, señala el camino a seguir, sin embargo, aún no se tiene claro, en virtud que, la posmodernidad decretó la muerte de la objetividad, en donde el hombre está saliendo de una era de certidumbre para adentrarse en la incertidumbre de un mundo lleno de situaciones complejas, donde pareciera no encontrar acomodo, y donde se busca formas y maneras de ubicarse sin conseguirlo.
Mucho se ha conversado acerca de la postmodernidad, los procesos educativos y culturales en donde se promueven ciertas transformaciones en las actividades académicas que en tiempos atrás consistían en una serie de actividades que tenían que ser casi siempre presenciales en el mismo espacio físico y en el mismo tiempo cronológico. Pero, actualmente, gracias a la aplicación y uso de las TICS, y su convergencia digital con diversas herramientas y estrategias, los nuevos procesos educativos y culturales se hacen más flexibles, globales y ubicuos, en virtud que ellos son independientes y están permanentemente disponibles del espacio físico y temporal desde donde se generan.

Desde esos cambios y/o transformaciones evidenciadas a lo largo de los últimos años, se observa que la tendencia actual en el aprendizaje está constituida por aspectos cognitivos y motivacionales, en un marco socializante, con el apoyo de las nuevas tecnologías de computación que se presentan como herramientas para la búsqueda del conocimiento. De esta manera, teorías como el conectivismo, engloban las ideas anteriores y señalan un cambio, aunque no muy claro todavía, para el aprendizaje a través de una educación multimodal aunado al hecho de incorporar una andragogía socio constructivista, donde se fomente el aprendizaje individual de manera socializada que pueda producir nuevos conocimientos con la participación de individuos en diversas latitudes.

Ahora bien, el hecho de utilizar e internalizar el uso de las herramientas TICS y su convergencia digital a través de estrategias educativas para facilitar los aprendizajes, no significa que todas las personas puedan acceder a la 
educación y a la cultura, en virtud que existen otros factores externos e internos en los cuales se debe profundizar para crear un aparataje tecnológico en torno a las concepciones educativas asistida por la tecnología, en virtud que a pesar de sus múltiples ventajas de conexión no sólo en el momento y en lugar que lo deseen, de acuerdo con sus deseos o necesidades informativas y cognitivas, y de los propios niveles de conocimientos y habilidades previas que se tengan, se evidencia la necesidad de una infraestructura social tecnológica que pueda acobijar y ofrecer nuevas maneras de enseñar y aprender. Desde este punto de vista, se considera importante que los docentes cumplan un rol que permita no solo conocer técnicamente las herramientas digitales educativas, sino saber aplicar de manera andragógica, para el logro de un acercamiento a pesar de la distancia a través de dichas tecnologías. De esta manera es más fácil adaptarla a los requerimientos de cada persona según sus intereses y gustos, y más allá de la formación que haya adquirido, o bien de la formación continua o permanente que pretenda seguir a lo largo de su vida.

En la era de la cultura digital en el ámbito educativo es preciso que todos los actores educativos tengan acceso a una formación básica en el uso de las TICS, de manera que puedan vivir y trabajar de forma natural en un contexto cada vez más apoyado en lo digital. Y aunque la propuesta es ir incorporando la enseñanza de las TICS en los planes educativos desde los primeros niveles de enseñanza (del mismo modo que integra la expresión oral, la lectura, escritura o el cálculo aritmético) para que así, en el futuro, el individuo se desenvuelva de una manera natural en un mundo digital, pero será necesario que las personas que ya hayan superado esos niveles puedan adquirir dichos conocimientos de manera autónoma e independiente, construyendo ellos mismos sus conocimientos nuevos. Por ello, los gobiernos y estados deben dar la posibilidad de adquirir una educación y cultura digital a todos los ciudadanos, desde este contexto, se deben unir esfuerzos en fomentar la adquisición de una infraestructura tecnológica fortalecida, desde los equipos de computadoras y todo lo relacionado con el hardware y con su respectivos programas software que permita constituir laboratorios computacionales centralizados, donde puedan converger los elementos necesarios para la construcción del conocimiento en redes.

Por otra parte, en la medida que los individuos avanzan en los niveles educativos, la cultura digital incide en el desarrollo de la capacidad para adquirir otros conocimientos o habilidades en el uso educativo de las TICS. En estos niveles es cuando el estudiante debe ir incorporando a su vida el idioma digital, a medida que va progresando académicamente. Así pues, del mismo modo que la mejor manera de aprender una lengua es vivir en una comunidad donde se habla ese idioma, para adquirir el idioma digital es preciso vivir en un hábitat digital, pero centrado en su aplicación con fines educativos donde pueda promover su aprendizaje a través de medios tecnológicos.

Por tanto, el objetivo final de la educación en la promoción de la cultura digital que se vaya cultivando desde la formación de los docentes, en virtud que serán los responsables de dirigir, asesorar y supervisar este nuevo proceso educativo, en donde se debe formar a todos los individuos para que sean capaces y más allá de la propia utilización que hagan de las 
TICS en sus actividades educativas o culturales, de aprehender con ellas mismas. En este sentido, la construcción del conocimiento debe entenderse como algo que se genera o se construye en todo momento, por cada individuo y de acuerdo con las necesidades o requerimientos cognitivos que tenga dicho individuo; y no tan sólo como un saber acumulado ya preexistente, el cual alguien se ha dedicado a prepararlo, ofrecerlo o presentarlo al que lo desee simplemente. La convergencia digital de las TICS en las universidades, con el apoyo del nuevo docente en el rol que asume deberá ayudar a que la construcción del conocimiento para prepararlo para la sociedad actual en la que se vive, por ello dicho objetivo sea más sencillo de conseguir, aun cuando las tecnologías han de ser entendidas más como un medio y no como un fin para lograrlo.

Desde esta perspectiva, en el campo educativo, en lo referente a la cultura digital, se puede decir que existe una doble valoración del uso de las TICS, en primera instancia, es que dicho uso, fomenta efectivamente la sistematización y la socialización del conocimiento, a través de un entorno educativo y cultural propio que facilita el trabajo cooperativo o colaborativo entre sus usuarios. En el segundo aspecto, es que, con la utilización de las TICS, a través del uso del Internet, se está favoreciendo la extensión y la profundización de muchos otros valores educativos y culturales como por ejemplo; la iniciativa, aprendizaje social, enseñanza social, convivencia, solidaridad, cooperación, colaboración, entre otros, en las personas que las utilizan. $Y$ es donde el docente debe ser garante del cumplimiento de estas normas y políticas de convivencia que permitan un buen clima socialtecnológico para el crecimiento afectivo entre los individuos.

Por ello, desde su aparición hasta su utilización en la vida social del ser humano, las TICS han encontrado en el campo de la educación y sobre todo en cultivarla cultura digital, un espacio de coincidencia muy favorable para su desarrollo, tanto en los procesos o tareas de enseñanza y aprendizaje (sea en ambientes o entornos presenciales, pero sobre todo virtuales y a distancia), como en aquellos o aquellas para el acceso al patrimonio cultural y artístico, o bien al saber científico y tecnológico y su respectiva divulgación.

\section{Referencias bibliográficas}

Bagozzi, D. (2020). Declaración conjunta del Director General de la OMC Roberto Azevêdo y el Director General de la OMS Tedros Adhanom Ghebreyesus. Organización Mundial de la Salud. https://www.who.int/ es/news/item/20-04-2020-jointstatement-by-wto-director-generalroberto-azev\%C3\%AAdo-and-whodirector-general-tedros-adhanomghebreyesus

Bartolomé Pina, A. (2008). Entornos de aprendizaje mixto en educación superior. RIED. Revista Iberoamericana De Educación a Distancia, 11(1), 15-51. https://doi. org/10.5944/ried.1.11.955

Bindé, J. (2005). Hacia las Sociedades del Conocimiento. UNESCO. Francia.

Cabero, J. (2014). La formación del profesorado en TIC: Modelo TPACK. Sevilla: Universidad de Sevilla

Camacho Marín, R., Rivas Vallejo, C., Gaspar Castro, M., \& Quiñonez Mendoza, C. (2020). Innovación y 
Chuquihuanca Yacsahuanca, Nelson; Pesantes Shimajuko, Soledad Marlene; Vásquez Rodríguez, Luis y Vargas De Olgado, Elena Cachicatari

Cultura digital en el contexto universitario: reflexiones valorativas en tiempos de pandemia

tecnología educativa en el contexto actual latinoamericano. Revista De Ciencias Sociales, 26, 460472. https://doi.org/10.31876/rcs. v26i0.34139

Covarrubias Hernández, Liliana. (2021). Educación a distancia: transformación de los aprendizajes. Telos: revista de Estudios Interdisciplinarios en Ciencias Sociales, 23(1), https://doi. org/10.36390/telos 231.12

Fourez, G. (2008). Cómo se elabora el conocimiento. Madrid: Ediciones Narcea. https://narceaediciones. es/es/educacion-hoy-estudios/126como-se-elabora-el-conocimiento-laepistemologia-desde-un-enfoque-so cioconstructivista-9788427715721. $\underline{\mathrm{html}}$

Freire, P. (1997). Pedagogía de la autonomía: saberes necesarios para la práctica educativa. Siglo XXI editores.

Gaonac'h, D., y Golder, C. (2005). Manual de psicología para la enseñanza. Siglo XXI.

García-Valcárcel, A. (coord.) (2008). Experiencias de innovación docente universitaria. I Congreso Internacional de Intercambio de Experiencias de Innovación Docente Universitaria. Universidad de Salamanca. https://dialnet.unirioja. es/servlet/libro?codigo $=400684$

Gardner, H. (2001). La Inteligencia Reformulada. Las inteligencias múltiples en el siglo XXI. Barcelona: Paidós. http://www.nodosele.com/ editorial

Jonnaert, P. (2002). Compétences et socioconstructivisme: Un cadre théorique. [Competencias y socioconstructivismo: un marco teórico]. Bruselas: De Boeck\&Larcier. Collection Perspectives en éducation et formation https://www.cairn.info/ competences-et-socioconstructivis me--9782804134587.htm

Knox, J.S. (2008). "Online Newspaper and TESOL Classrooms: a Multimodal Perspective". En L. Unsworth (Ed.), Multimodal Semiotics. Functional Analysis in Contexts of Education (pp. 139-158). Londres: Continuum

Kress, G. (2010). Multimodality. A Social Semiotic Approach to Contemporary Communication. Londres: Routledge.

Luengo, J., Luzón, A. y Torres, M. (2008). Las reformas educativas basadas en el enfoque por competencias: una visión comparada. Profesorado. Universidad de Granada. http://www. ugr.es/local/recfpro/rev123ed.pdf

Lyotard, J.F. (1989). ¿Por qué filosofar? Paidós.

Lyotard, J.F. (2004). La condición posmoderna. Cátedra.

Mayer, R. (2009). Multimedia Learning. Cambridge University Press.

Medina, J. (2006). Visión compartida de futuro. Programa Editorial Universidad del Valle, Colombia. http://programaeditorialunivalle.com/ libro-vision-compartida-de-futuroeconomia.html

Miller, R. (2011). Vygotsky in Perspective. Cambridge: Cambridge University Press.

Morales, E. M. (2010). Gestión del conocimiento en sistemas E-learning, basado en objetos de aprendizaje, cualitativa y pedagógicamente definidos. Salamanca: Ediciones Universidad de Salamanca. https:// redined.mecd.gob.es/xmlui/ handle/11162/179131

Perkins, D. (2003). ¿Cómo hacer el pensamiento visible?. http:// conexiones.dgire.unam.mx/wpcontent/uploads/2017/11/perkins 
david como hacer visible el pensamiento.pdf

Pritchard, A. (2013). Ways of Learning: Learning theories and learning styles in the classroom. Routledge Taylor y Francis Group. http://www. epitropakisg.gr/grigorise/ways $\% 20$ of\%20learning.pdf

Salinas, J., Darder, A., y De Benito, B. (2015). Las TIC en la enseñanza superior: e-learning, b-learning y m-learning. En Cabero, J. y Barroso, L. , Nuevos Retos en Tecnología Educativa (p:153-173). Madrid: Síntesis

Santamaría F. (2006). Conectivismo: Una teoría del aprendizaje para la era digital. https://www.comenius. $\mathrm{cl} / \mathrm{recursos} / \mathrm{virtual/minsal} \mathrm{v} 2 /$ Modulo 1/Recursos/Lectural conectivismo Siemens.pdf

Serrano, J. y Pons, R. M. (2011). El Constructivismo hoy: enfoques constructivistas en educación.
Revista Electrónica Científica Scielo REDIE, 13(1). https://bit.ly/3dofdrc

Siemens, G. (2006). Conectivism: learning and knowledge today [enlínea]. http://www.educationau. edu.au/jahia/webdav/site/ myjahiasite/shared/globalsummit/ gs2006 siemens.pdf

Suárez, J., Almerich, G., Gargallo, B. , y Aliaga, F. (2010). Las competencias en TIC del Profesorado y su relación con el uso de los recursos tecnológicos. Archivos Analíticos de Políticas Educativas, 18(1). https://dialnet.unirioja.es/servlet/ articulo?codigo $=3199091$

Wertsch, J. (1988). Vygotsky y la formación social de la mente. Paidos. https://www.planetadelibros.com/ libro-vygotsky-y-la-formacion-socialde-la-mente/18810

Wiske, M. S. (1999). La Enseñanza para la Comprensión. Vinculación entre la investigación y la práctica. Argentina: Paidós. https://bit.ly/3xZYIPq 